\title{
COUNTING MULTIPLICATIVE GROUPS WITH PRESCRIBED SUBGROUPS
}

\author{
JENNA DOWNEY AND GREG MARTIN
}

\begin{abstract}
We examine two counting problems that seem very group-theoretic on the surface but, on closer examination, turn out to concern integers with restrictions on their prime factors.

First, given an odd prime $q$ and a finite abelian $q$-group $H$, we consider the set of integers $n \leq x$ such that the Sylow $q$-subgroup of the multiplicative group $(\mathbb{Z} / n \mathbb{Z})^{\times}$is isomorphic to $H$. We show that the counting function of this set of integers is asymptotic to $K x(\log \log x)^{\ell} /(\log x)^{1 /(q-1)}$ for explicit constants $K$ and $\ell$ depending on $q$ and $H$.

Second, we consider the set of integers $n \leq x$ such that the multiplicative group $(\mathbb{Z} / n \mathbb{Z})^{\times}$is "maximally non-cyclic", that is, such that all of its prime-power subgroups are elementary groups. We show that the counting function of this set of integers is asymptotic to $A x /(\log x)^{1-\xi}$ for an explicit constant $A$, where $\xi$ is Artin's constant.

As it turns out, both of these group-theoretic problems can be reduced to problems of counting integers with restrictions on their prime factors, allowing them to be addressed by classical techniques of analytic number theory.
\end{abstract}

\section{INTRODUCTION}

Counting problems for integers with restrictions on their prime factors have been a topic of interest to number theorists for many years, with squarefree numbers and friable numbers (integers without large prime factors) being typical examples. Particularly relevant is Landau's investigation [4] of the counting function of those integers $n$ expressible as the sum of two squares; thanks to the classical characterization of Fermat, this property is equivalent to a restriction on the prime factors of $n$ that are congruent to $3(\bmod 4)$. Similar ideas allowed Ford, Luca, and Moree [3] to count the integers $n \leq x$ such that the Euler totient function $\phi(n)$ is not divisible by a fixed prime $q$, or equivalently such that $q^{2}$ does not divide $n$ and no prime divisor of $n$ is congruent to $1(\bmod q)$.

This last result can be restated as counting the integers $n$ up to $x$ for which the Sylow $q$-subgroup of $(\mathbb{Z} / n \mathbb{Z})^{\times}$is the trivial group. And indeed, examining group-theoretic statistics of the family of multiplicative groups $(\mathbb{Z} / n \mathbb{Z})^{\times}$is a fertile source of problems of interest to analytic number theorists, starting directly with the distribution of the cardinality $\phi(n)$. For example, the length of the invariant factor decomposition of $(\mathbb{Z} / n \mathbb{Z})^{\times}$(see Section 3 for the definition) is essentially the number of distinct prime factors of $n$, and so these lengths satisfy an Erdôs-Kac law (they are asymptotically normally distributed when suitably normalized). Recent work of the second author with Chang [1] and Troupe [5] examined, respectively, the counting function of those integers with a prescribed least invariant factor and an Erdôs-Kac law for the total number of subgroups of $(\mathbb{Z} / n \mathbb{Z})^{\times}$.

When speaking on the work [5] at the 2017 Alberta Number Theory Days, Lee Troupe was asked by Colin Weir if it was possible to count, for a fixed prime $q$ and a fixed finite abelian $q$-group $H$, the number of integers $n \leq x$ for which the Sylow $q$-subgroup of $(\mathbb{Z} / n \mathbb{Z})^{\times}$is isomorphic to $H$,

2010 Mathematics Subject Classification. 11N25, 11N37, 11N45, 11N64, $20 \mathrm{~K} 01$. 
thus generalizing the aforementioned result of Ford, Luca, and Moree. Answering this question is the main focus of this paper; we establish some notation to describe our results.

Definition 1.1. Let $\mathbb{Z}_{n}=(\mathbb{Z} / n \mathbb{Z})^{+}$and $\mathbb{Z}_{n}^{\times}=(\mathbb{Z} / n \mathbb{Z})^{\times}$denote the additive group and multiplicative group, respectively, of the quotient ring $\mathbb{Z} / n \mathbb{Z}$. For any prime $q$, let $G_{q}(n)$ denote the Sylow $q$-subgroup of $\mathbb{Z}_{n}^{\times}$, that is, the unique subgroup of $\mathbb{Z}_{n}^{\times}$whose cardinality is the highest power of $q$ that divides $\phi(n)$.

Definition 1.2. For any finite abelian $q$-group $H$, let $D(H, x)=\#\left\{n \leq x: G_{q}(n) \cong H\right\}$.

Because our asymptotic formula for $D(H, x)$ will depend upon $H$, we need a standard notation for isomorphism classes of finite abelian $q$-groups. From the classification of finite abelian groups, every abelian group of prime-power order can be labeled by a partition (a nonincreasing sequence of positive integers).

Definition 1.3. Given a partition $\boldsymbol{\alpha}=\left(\alpha_{1}, \ldots, \alpha_{j}\right)$, denote its length by $\ell(\boldsymbol{\alpha})=j$. Let $\mathbb{Z}_{q^{\alpha}}$ denote the finite abelian $q$-group $\mathbb{Z}_{q^{\alpha_{1}}} \times \mathbb{Z}_{q^{\alpha_{2}}} \times \cdots \times \mathbb{Z}_{q^{\alpha_{j}}}$.

The following theorem, which we prove in Section 2, gives an asymptotic formula for the number of integers $n \leq x$ for which the Sylow $q$-subgroup of $\mathbb{Z}_{n}^{\times}$is isomorphic to any particular finite abelian $q$-group $\mathbb{Z}_{q^{\alpha}}$.

Theorem 1.4. Let $q$ be an odd prime and $\boldsymbol{\alpha}$ a partition. Then

$$
D\left(\mathbb{Z}_{q^{\boldsymbol{\alpha}}}, x\right)=K\left(\mathbb{Z}_{q^{\boldsymbol{\alpha}}}\right) \frac{x(\log \log x)^{\ell(\boldsymbol{\alpha})}}{(\log x)^{1 /(q-1)}}\left(1+O_{q, \boldsymbol{\alpha}}\left(\frac{1}{\log \log x}\right)\right),
$$

where $K\left(\mathbb{Z}_{q^{\alpha}}\right)$ is the constant from Definition 2.29 below.

This result does in fact cover the case where $\boldsymbol{\alpha}$ is the empty partition, so that $\mathbb{Z}_{q^{\alpha}}$ is the trivial group; consequently, one special case of Theorem 1.4 is the result of Ford, Luca, and Moree [3] mentioned above.

Corollary 1.5. Let $q$ be an odd prime. The number of $n \leq x$ for which $q \nmid \phi(n)$ equals

$$
D\left(\mathbb{Z}_{q^{\emptyset}}, x\right)=\frac{B_{q} x}{(\log x)^{1 /(q-1)}}\left(1+O_{q}\left(\frac{1}{\log \log x}\right)\right),
$$

where $B_{q}$ is the constant depending on q from Definition 2.5 below.

The methods of this paper could in principle handle the variant of Theorem 1.4 where $q=2$, but we do not do so herein. (Note that the analogous variant of Corollary 1.5 is trivial, since $2 \mid \phi(n)$ for all $n \geq 3$; this fact would necessitate a somewhat different starting point for the $q=2$ variant of Theorem 1.4, even though the subsequent procedure would be very similar.)

In Section 3 we shift our focus to a problem involving the global structure of the multiplicative group $\mathbb{Z}_{n}^{\times}$. The motivation for our next theorem comes from the fact that it is easy to count the integers $n \leq x$ for which $\mathbb{Z}_{n}^{\times}$is cyclic: these are precisely the integers possessing primitive roots (namely $1,2,4$, and $p^{r}$ and $2 p^{r}$ for odd primes $p$ and integers $r \geq 1$ ) and thus have counting function asymptotic to $\operatorname{li}(x)+\operatorname{li}\left(\frac{x}{2}\right) \sim \frac{3}{2} x / \log x$ by the prime number theorem. We might ask for the opposite extreme: what is the "least cyclic" that a finite abelian group can be, and how prevalent are such groups in the family of multiplicative groups?

Several notions of such a group being "maximally non-cyclic" turn out to be mutually equivalent (see Definition 3.1 below); one way to describe a maximally non-cyclic finite abelian group is one 
all of whose Sylow $q$-subgroups are elementary groups (direct products of copies of $\mathbb{Z}_{q}$ ). The counting function for the corresponding integers $n$ turns out to be quite interesting:

Theorem 1.6. The number of integers $n$ up to $x$ such that $\mathbb{Z}_{n}^{\times}$is maximally non-cyclic is asymptotic to $A x /(\log x)^{1-\xi}$, where $\xi$ is Artin's constant and $A$ is the constant in Definition 3.3 below.

The proof gives a relative error of $1 /(\log x)^{1-\varepsilon}$ for any $\varepsilon>0$. We point out that the main term contains an exponent of $\log x$ that is (presumably) irrational, which is an unusual feature of an asymptotic formula arising from a reasonably natural property of $\mathbb{Z}_{n}^{\times}$.

\section{MultipliCATIVE GROUPS With A PRESCRIBEd Sylow $q$-SUbGROUP}

Throughout this paper, we will use the letters $p$ (with or without subscripts), $q$, and $t$ exclusively to denote primes.

2.1. Reduction to analytic number theory. In this section we convert the group-theoretic property $G_{q}(n)=H$ into specific constraints on the primes dividing $n$, so that the problem of counting multiplicative groups $\mathbb{Z}_{n}^{\times}$with specified Sylow $q$-subgroup $H$ is converted into a nested sum indexed by a particular factorization of $n$ (see Proposition 2.4). Since the power of $q$ itself that divides $n$ affects the Sylow $q$-subgroup of $\mathbb{Z}_{n}^{\times}$in a particular way, we stratify the integers according to that power and count each stratum of integers separately.

Definition 2.1. For a prime $q$ and a nonzero integer $x$, define $\nu_{q}(x)$ to be the largest nonnegative integer $k$ such that $q^{k}$ divides $x$.

Definition 2.2. Given a prime $q$, a finite abelian $q$-group $H$, and a nonnegative integer $k$, define $D_{k}(H, x)=\#\left\{n \leq x: \nu_{q}(n)=k, G_{q}(n)=H\right\}$. Note that $D(H, x)=\sum_{k=0}^{\infty} D_{k}(H, x)$, where $D(H, x)$ is as in Definition 1.2 .

We will show in Lemma 2.28 that $D_{k}(H, x)$, if nonzero, is equal to $D_{0}\left(\mathbb{Z}_{H^{\prime}}, x\right)$ for a certain $q$ group $H^{\prime}$ depending on $H$ and $k$. Therefore our main technical goal in Section 2 is to obtain an asymptotic formula for $D_{0}(H, x)$.

To avoid requiring notation for the lengths of partitions, we can regard partitions as infinite nonincreasing sequences of nonnegative integers with only finitely many positive terms.

Definition 2.3. For any partition $\boldsymbol{\alpha}=\left(\alpha_{1}, \alpha_{2}, \ldots\right)$, the conjugate partition $\boldsymbol{a}=\left(a_{1}, a_{2}, \ldots\right)$ of $\left(\alpha_{1}, \alpha_{2}, \ldots\right)$ is the partition whose Ferrers diagram is the transpose of the Ferrers diagram of $\left(\alpha_{1}, \alpha_{2}, \ldots\right)$, so that $a_{j}=\#\left\{k: \alpha_{k} \geq j\right\}$ for all $j \geq 1$; in particular, $a_{j}-a_{j+1}=\#\left\{k: \alpha_{k}=j\right\}$. Using this conjugate partition, define

$$
C(\boldsymbol{\alpha})=\prod_{u=1}^{\infty} \frac{1}{\left(a_{u}-a_{u+1}\right) !}=\prod_{u=1}^{\alpha_{1}} \frac{1}{\left(a_{u}-a_{u+1}\right) !} .
$$

Proposition 2.4. For any odd prime $q$ and any partition $\boldsymbol{\alpha}=\left(\alpha_{1}, \ldots, \alpha_{j}\right)$,

$$
\begin{aligned}
& D_{0}\left(\mathbb{Z}_{q^{\alpha}}, x\right) \\
& =C(\boldsymbol{\alpha}) \sum_{\substack{p_{1} \leq x / 3 \\
\nu_{q}\left(p_{1}-1\right)=\alpha_{1}}} \sum_{\substack{p_{2} \leq x / 3 p_{1} \\
p_{2} \neq p_{1} \\
\nu_{q}\left(p_{2}-1\right)=\alpha_{2}}} \cdots \sum_{\begin{array}{c}
p_{j-1} \leq x / 3 p_{1} \cdots p_{j-2} \\
p_{j-1} \neq p_{1}, \ldots, p_{j-2} \\
\nu_{q}\left(p_{j-1}-1\right)=\alpha_{j-1}
\end{array}} \sum_{\begin{array}{c}
p_{j} \leq x / p_{1} \cdots p_{j-1} \\
p_{j} \neq p_{1}, \ldots, p_{j-1} \\
\nu_{q}\left(p_{j}-1\right)=\alpha_{j}
\end{array} \quad(t \mid m \text { and } t \equiv 1(\bmod q)) \Rightarrow t \in\left\{p_{1}, \ldots, p_{j}\right\}}
\end{aligned}
$$

where $t$ denotes a generic prime factor of $m$. Here, $C(\boldsymbol{\alpha})$ is the constant defined in equation (1). 
Proof. We can write $n$ as the product of primes $n=2^{\beta} p_{1}^{\beta_{1}} p_{2}^{\beta_{2}} \cdots p_{k}^{\beta_{k}}$ where $\beta \geq 0, \beta_{1}, \beta_{2}, \ldots, \beta_{k}>$ 0 and $q \neq p_{j}$ for each $1 \leq j \leq k$. By the Chinese remainder theorem

$$
\begin{aligned}
\mathbb{Z}_{n}^{\times} & \cong \mathbb{Z}_{2^{\beta}}^{\times} \times\left(\mathbb{Z}_{p_{1}^{\beta_{1}-1}} \times \mathbb{Z}_{p_{1}-1}\right) \times\left(\mathbb{Z}_{p_{2}^{\beta_{2}-1}} \times \mathbb{Z}_{p_{2}-1}\right) \times \cdots \times\left(\mathbb{Z}_{p_{k}^{\beta_{t}-1}} \times \mathbb{Z}_{p_{k}-1}\right) \\
& \cong\left(\mathbb{Z}_{2^{\beta}}^{\times} \times \mathbb{Z}_{p_{1}^{\beta_{1}-1}} \times \mathbb{Z}_{p_{2}^{\beta_{2}-1}} \times \cdots \times \mathbb{Z}_{p_{t}^{\beta_{k}-1}}\right) \times\left(\mathbb{Z}_{p_{1}-1} \times \mathbb{Z}_{p_{2}-1} \times \cdots \times \mathbb{Z}_{p_{k}-1}\right) .
\end{aligned}
$$

Since $q$ is an odd prime not dividing $n$, we see that $q$ does not divide the cardinality of the first factor; therefore the Sylow $q$-subgroup of $\mathbb{Z}_{n}^{\times}$is the same as the Sylow $q$-subgroup of the second

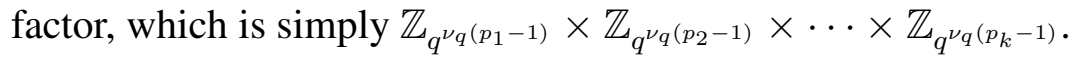

It follows that $G_{q}(n)=\mathbb{Z}_{q^{\alpha}}$ if and only if the multisets $\boldsymbol{\alpha}$ and $\left\{\nu_{q}\left(p_{i}-1\right): 1 \leq i \leq k\right\}$ are the same except for occurrences of 0 in the latter multiset, that is, if and only if $n$ has, for every integer $u \geq 1$, exactly $a_{u}-a_{u+1}$ distinct prime factors $p$ satisfying $\nu_{q}(p)=\alpha_{u}$.

Now set $j=\ell(\boldsymbol{\alpha})$ and consider the expression

$$
\sum_{\substack{p_{1} \leq x \\
\nu_{q}\left(p_{1}-1\right)=\alpha_{1}}} \sum_{\substack{p_{2} \leq x / p_{1} \\
p_{2} \neq p_{1} \\
\nu_{q}\left(p_{2}-1\right)=\alpha_{2}}} \sum_{\substack{p_{j} \leq x / p_{1} \cdots p_{j-1} \\
p_{j} \neq p_{1}, \ldots, p_{j-1} \\
\nu_{q}\left(p_{j}-1\right)=\alpha_{j}}} \sum_{\left.\begin{array}{c}
m \leq x / p_{1} \cdots p_{j} \\
q \nmid m \\
(t \mid m
\end{array} \text { and } t \equiv 1(\bmod q)\right) \Rightarrow t \in\left\{p_{1}, \ldots, p_{j}\right\}} 1 .
$$

This expression counts integers of the form $n=p_{1} p_{2} \cdots p_{j} m$, where $p_{1}, \ldots, p_{j}$ are distinct primes such that $\left\{\nu_{q}\left(p_{i}-1\right): 1 \leq i \leq j\right\}=\boldsymbol{\alpha}$ as multisets and $\nu_{q}(p-1)=0$ for every $p \mid m$. In other words, it counts integers $n$ such that $G_{q}(n)=\mathbb{Z}_{q^{\alpha}}$, except that it counts such integer with multiplicity because $\left(p_{1}, \ldots, p_{j}\right)$ is an ordered tuple: for each $u \geq 1$ we may arbitrarily permute the $a_{u}-a_{u+1}$ primes $p_{i}$ in the tuple that satisfy $\nu_{q}\left(p_{i}\right)=\alpha_{u}$ and still obtain the same $n$. Consequently we must divide by $\left(a_{u}-a_{u+1}\right)$ ! for each $u \geq 1$ to compensate for this multiple counting, which is the same as multiplying the expression (3) by $C(\boldsymbol{\alpha})$.

Finally, if $p_{i}$ is greater than $x / 3 p_{1} \cdots p_{i-1}$ for any $1 \leq i \leq j-1$, then the sum over $p_{i+1}$ in the expression (3) is empty, and therefore we may alter the upper bounds of summation accordingly, reaching the expression in equation (2) as desired.

2.2. Application of the Selberg-Delange method. Proposition 2.4 provides a clear relationship between the original problem of counting prescribed Sylow $q$-subgroups and the more analytic problem of counting integers with restrictions on their prime factors. The innermost sum in equation (2), in particular, is exactly of this latter type, and thus can be successfully estimated by the Selberg-Delange method. We cite an application of this technique from [1] that has been tailored to this purpose.

Definition 2.5. For any odd prime $q$ and any prime $p \neq q$, let $k_{p}$ denote the multiplicative order of $p$ modulo $q$. Then define

$$
B_{q}=\frac{1}{\Gamma(1-1 /(q-1))}\left(1-\frac{1}{q}\right)^{1-1 /(q-1)} \prod_{\substack{p \neq q \\ p \neq 1(\bmod q)}}\left(1-\frac{1}{p^{k_{p}}}\right)^{-1 / k_{p}} \prod_{\chi \neq \chi_{0}} L(1, \chi)^{-1 /(q-1)}
$$

where $\Gamma(z)$ is the classical Gamma function. 
Proposition 2.6. Let $q$ be an odd prime, and let $p_{1}, \ldots, p_{j}$ be distinct prime numbers congruent to $1(\bmod q)$. For $y \geq 3$,

$$
\sum_{\substack{m \leq y \\ q \nmid m \\(t \mid m \text { and } t \equiv 1(\bmod q)) \Rightarrow t \in\left\{p_{1}, \ldots, p_{j}\right\}}} 1=\frac{B_{q} y}{(\log y)^{1 /(q-1)}} \prod_{i=1}^{j}\left(1-\frac{1}{p_{i}}\right)^{-1}+O_{j}\left(\frac{y}{(\log y)^{1+1 /(q-1)}}\right),
$$

where $t$ denotes a generic prime factor of $m$.

Proof. Define unions of residue classes

$$
\mathcal{B}=\{n \not \equiv 0,1(\bmod q)\} \quad \text { and } \quad \mathcal{B}^{\prime}=\{n \equiv 1(\bmod q)\}
$$

so that we are trying to count integers whose prime factors all lie in $\mathcal{B} \cup\left\{p_{1}, \ldots, p_{j}\right\}$.

We begin by quoting [1, Theorem 3.6] with the set $\mathcal{B}$ just defined, so that (using Notation 3.1 from that paper) $B=q-2$ and $\underline{B}=1$ and $\beta=1-1 /(q-1)$; we also set $\mathcal{I}=\left\{p_{1}, \ldots, p_{j}\right\}$ and $\mathcal{R}=\emptyset$. Since we are allowing our error terms to depend on $q$, we may simplify the error term from [1, Theorem 3.6], and we may also ignore the assumption that $\log y \geq \alpha q^{1 / 2} \log ^{2} q$. The conclusion (remembering that $t$ denotes a generic prime) is that

$$
\begin{aligned}
\#\{m \leq y: q \nmid m & \left.,(t \mid m \text { and } t \equiv 1(\bmod q)) \Rightarrow t \in\left\{p_{1}, \ldots, p_{j}\right\}\right\} \\
& =\frac{y}{(\log y)^{1 /(q-1)}}\left(\frac{G_{\mathcal{B}}(1)}{\Gamma(1-1 /(q-1))} \prod_{i=1}^{j}\left(1-\frac{1}{p_{i}}\right)^{-1}+O\left(2^{j}(\log y)^{-1}\right)\right) .
\end{aligned}
$$

It thus remains to evaluate $G_{\mathcal{B}}(1)$.

In the proof of [1, Proposition 4.1], where the set $\mathcal{B}^{\prime}$ is denoted as $\{1\}$, it is shown that

$$
G_{\mathcal{B}^{\prime}}(1)=\left(\frac{\phi(q)}{q} \prod_{\substack{\chi(\bmod q) \\ \chi \neq \chi_{0}}} L(1, \chi)\right)^{1 / \phi(q)} \prod_{\substack{p \nmid q \\ p \neq \equiv 1(\bmod q)}}\left(1-\frac{1}{p^{\operatorname{ord}_{q}(p)}}\right)^{1 / \operatorname{ord}_{q}(p)} .
$$

Moreover, [1, Remark 3.5] tells us that $G_{\mathcal{B}}(1) G_{\mathcal{B}^{\prime}}(1)=\phi(q) / q$. Therefore

$$
\begin{aligned}
G_{\mathcal{B}}(1)=\frac{\phi(q)}{q G_{\mathcal{B}^{\prime}}(1)} & =\frac{\phi(q)}{q}\left(\frac{\phi(q)}{q} \prod_{\substack{\chi(\bmod q) \\
\chi \neq \chi_{0}}} L(1, \chi)\right)^{-1 / \phi(q)} \prod_{\substack{p \nmid q \\
p \neq 1(\bmod q)}}\left(1-\frac{1}{p^{\operatorname{ord}_{q}(p)}}\right)^{-1 / \operatorname{ord}_{q}(p)} \\
& =\left(1-\frac{1}{q}\right)^{1-1 /(q-1)} \prod_{\substack{\chi(\bmod q) \\
\chi \neq \chi_{0}}} L(1, \chi)^{-1 /(q-1)} \prod_{\substack{p \neq q \\
p \neq 1(\bmod q)}}\left(1-\frac{1}{p^{\operatorname{ord}_{q}(p)}}\right)^{-1 / \operatorname{ord}_{q}(p)}
\end{aligned}
$$

which, together with the Gamma factor from equation (4), equals $B_{q}$ as given in Definition 2.5.

2.3. Technical lemmas. Motivated by the expressions that will appear when we apply Proposition 2.6 to equation (2), we now establish a collection of technical lemmas that will be used in the next section to prove the important Proposition 2.20, That result will subsequently allow us to establish the recursive Propositions 2.22 and 2.24, which will provide an evaluation of the iterated sum in equation (2). Though many of the techniques of this section are standard, we do highlight the use of the following hypergeometric function as a tool for evaluating certain sums over primes with fractional powers of a logarithm (see Lemmas 2.12 and 2.14). 
Definition 2.7. For any $\gamma \in \mathbb{R} \backslash \mathbb{N}$, define

$$
H_{\gamma}(z)=-\sum_{n=1}^{\infty} \frac{\gamma}{n-\gamma} z^{n}
$$

Note that the power series defining $H_{\gamma}(z)$ converges for $|z|<1$ by the ratio test. (One could also define $H_{\gamma}(z)=-\gamma \Phi(z, 1,-\gamma)-1$ where $\Phi$ is the Hurwitz-Lerch transcendent.)

Lemma 2.8. Let $\gamma>0$ such that $\gamma \notin \mathbb{N}$.

(a) For $0 \leq z \leq \frac{1}{2}$, we have $H_{\gamma}(z) \ll_{\gamma} z$.

(b) For $0 \leq z<1$, we have $H_{\gamma}(z)=\gamma \log (1-z)+O_{\gamma}(1)$.

Proof. Part (a) follows simply from the fact that $H_{\gamma}$ is analytic on a neighborhood of $\left[0, \frac{1}{2}\right]$ and $H_{\gamma}(0)=0$. Using Definition 2.7 and the power series for $\log (1-z)$,

$$
\begin{aligned}
H_{\gamma}(z)-\gamma \log (1-z) & =-\sum_{n=1}^{\infty} \frac{\gamma z^{n}}{n-\gamma}-\gamma \sum_{n=1}^{\infty} \frac{-z^{n}}{n} \\
& =-\gamma^{2} \sum_{n=1}^{\infty} \frac{z^{n}}{n(n-\gamma)} \ll_{\gamma} \sum_{n=1}^{\infty} \frac{1}{n|n-\gamma|} \ll_{\gamma} 1,
\end{aligned}
$$

which establishes part (b).

Lemma 2.9. Let $\gamma>0$ such that $\gamma \notin \mathbb{N}$, and let $x>1$. For $0<z<1$,

$$
\frac{d}{d z}\left(\frac{H_{\gamma}(z)}{\gamma(z \log x)^{\gamma}}\right)=-\frac{1}{(1-z)(z \log x)^{\gamma}} .
$$

Proof. We differentiate the power series in Definition 2.7 term by term to obtain

$$
\begin{aligned}
\frac{d}{d z}\left(\frac{H_{\gamma}(z)}{\gamma(z \log x)^{\gamma}}\right) & =\frac{d}{d z}\left(\frac{-\sum_{n=1}^{\infty} \frac{\gamma}{n-\gamma} z^{n}}{\gamma(z \log x)^{\gamma}}\right) \\
& =\frac{-\gamma(z \log x)^{\gamma} \sum_{n=1}^{\infty} \frac{n \gamma}{n-\gamma} z^{n-1}+\gamma^{2}(\log x)^{\gamma} z^{\gamma-1} \sum_{n=1}^{\infty} \frac{\gamma}{n-\gamma} z^{n}}{\gamma^{2}(z \log x)^{2 \gamma}} \\
& =\frac{-\gamma^{2}(\log x)^{\gamma} z^{\gamma}\left(\sum_{n=1}^{\infty} \frac{n}{n-\gamma} z^{n-1}-\sum_{n=1}^{\infty} \frac{\gamma}{n-\gamma} z^{n-1}\right)}{\gamma^{2}(z \log x)^{2 \gamma}} \\
& =\frac{-\sum_{n=1}^{\infty} z^{n-1}}{(z \log x)^{\gamma}}=-\frac{1}{(1-z)(z \log x)^{\gamma}}
\end{aligned}
$$

as desired.

Lemma 2.10. Let $\gamma>0$ such that $\gamma \notin \mathbb{N}$, and let $x>1$. For $1<u<x$,

$$
\frac{d}{d u}\left(\frac{H_{\gamma}\left(1-\frac{\log u}{\log x}\right)}{\gamma(\log (x / u))^{\gamma}}\right)=\frac{1}{(u \log u)(\log (x / u))^{\gamma}} .
$$

Proof. Using the change of variables $z=1-(\log u) / \log x$, so that $z \log x=\log (x / u)$ and $\frac{d z}{d u}=-1 /(u \log x)$, we have

$$
\frac{d}{d u}\left(\frac{H_{\gamma}\left(1-\frac{\log u}{\log x}\right)}{\gamma(\log (x / u))^{\gamma}}\right)=\frac{d}{d z}\left(\frac{H_{\gamma}(z)}{\gamma(z \log x)^{\gamma}}\right)\left(-\frac{1}{u \log x}\right) .
$$


The assumption $1<u<x$ implies that $0<z<1$, and so by Lemma 2.9 ,

$$
\begin{aligned}
\frac{d}{d u}\left(\frac{H_{\gamma}\left(1-\frac{\log u}{\log x}\right)}{\gamma(\log (x / u))^{\gamma}}\right) & =-\frac{1}{(1-z)(z \log x)^{\gamma}}\left(-\frac{1}{u \log x}\right) \\
& =\frac{1}{((\log u) / \log x)(\log (x / u))^{\gamma}} \frac{1}{u \log x}
\end{aligned}
$$

as desired.

Lemma 2.11. Let $\gamma>0$. For $y \geq 4$,

$$
\int_{2}^{\sqrt{y}} \frac{1}{(u \log u)(\log (y / u))^{\gamma}} d u=\frac{\log \log y}{(\log y)^{\gamma}}+O_{\gamma}\left(\frac{1}{(\log y)^{\gamma}}\right) .
$$

Proof. Since $u \leq \sqrt{y}$ in the integrand, we may write

$$
\begin{aligned}
(\log (y / u))^{-\gamma} & =(\log y)^{-\gamma}\left(1-\frac{\log u}{\log y}\right)^{-\gamma} \\
& =(\log y)^{-\gamma}\left(1+O_{\gamma}\left(\frac{\log u}{\log y}\right)\right)=(\log y)^{-\gamma}+O_{\gamma}\left((\log y)^{-\gamma-1} \log u\right),
\end{aligned}
$$

and therefore

$$
\begin{aligned}
\int_{2}^{\sqrt{y}} & \frac{1}{(u \log u)(\log (y / u))^{\gamma}} d u \\
& =(\log y)^{-\gamma} \int_{2}^{\sqrt{y}} \frac{1}{u \log u} d u+O_{\gamma}\left((\log y)^{-\gamma-1} \int_{2}^{\sqrt{y}} \frac{\log u}{u \log u} d u\right) \\
& =(\log y)^{-\gamma}(\log \log \sqrt{y}-\log \log 2)+O_{\gamma}\left((\log y)^{-\gamma-1}(\log \sqrt{y}-\log 2)\right)
\end{aligned}
$$

which implies the statement of the lemma since $\log \log \sqrt{y}=\log \log y+O(1)$.

Lemma 2.12. Let $\gamma>0$ such that $\gamma \notin \mathbb{N}$, let $q$ be prime, and let $\alpha \in \mathbb{N}$. For $y \geq 4$,

$$
\sum_{\substack{p \leq \sqrt{y} \\ \nu_{q}(p-1)=\alpha}} \frac{1}{p(\log (y / p))^{\gamma}}=\frac{\log \log y}{q^{\alpha}(\log y)^{\gamma}}+O_{\gamma}\left(\frac{1}{(\log y)^{\gamma}}\right) .
$$

Proof. If we define

$$
M(x)=\sum_{\substack{p \leq x \\ \nu_{q}(p-1)=\alpha}} \frac{1}{p}=\sum_{\substack{p \leq x \\ p \equiv 1\left(\bmod q^{\alpha}\right)}} \frac{1}{p}-\sum_{\substack{p \leq x \\ p \equiv 1\left(\bmod q^{\alpha+1}\right)}} \frac{1}{p}
$$

then a Mertens-type formula for arithmetic progressions [6, Corollary 4.12] shows that there exist constants $c_{q^{\alpha}}$ and $c_{q^{\alpha+1}}$ such that

$$
\begin{aligned}
M(x) & =\left(\frac{\log \log x}{\phi\left(q^{\alpha}\right)}+c_{q^{\alpha}}+O\left(\frac{1}{\log x}\right)\right)-\left(\frac{\log \log x}{\phi\left(q^{\alpha+1}\right)}+c_{q^{\alpha+1}}+O\left(\frac{1}{\log x}\right)\right) \\
& =\frac{\log \log x}{q^{\alpha}}+c_{q^{\alpha}}-c_{q^{\alpha+1}}+O\left(\frac{1}{\log x}\right)
\end{aligned}
$$


for $x \geq 2$. Setting

$$
R(x)=M(x)-\left(\frac{\log \log x}{q^{\alpha}}+c_{q^{\alpha}}-c_{q^{\alpha+1}}\right) \ll \frac{1}{\log x},
$$

it follows that

$$
\begin{aligned}
\sum_{\substack{p \leq \sqrt{y} \\
\nu_{q}(p-1)=\alpha}} \frac{1}{p(\log (y / p))^{\gamma}} & =\int_{2}^{\sqrt{y}} \frac{1}{(\log (y / u))^{\gamma}} d M(u) \\
& =\int_{2}^{\sqrt{y}} \frac{1}{(\log (y / u))^{\gamma}} d\left(\frac{\log \log u}{q^{\alpha}}+c_{q^{\alpha}}-c_{q^{\alpha+1}}+R(u)\right) \\
& =\frac{1}{q^{\alpha}} \int_{2}^{\sqrt{y}} \frac{1}{(\log (y / u))^{\gamma}} \frac{d u}{u \log u}+\int_{2}^{\sqrt{y}} \frac{1}{(\log (y / u))^{\gamma}} d R(u) \\
& =\frac{1}{q^{\alpha}}\left(\frac{\log \log y}{(\log y)^{\gamma}}+O_{\gamma}\left(\frac{1}{(\log y)^{\gamma}}\right)\right)+\int_{2}^{\sqrt{y}} \frac{1}{(\log (y / u))^{\gamma}} d R(u)
\end{aligned}
$$

by Lemma 2.11. On the other hand, integrating by parts yields

$$
\begin{aligned}
\int_{2}^{\sqrt{y}} \frac{1}{(\log (y / u))^{\gamma}} d R(u) & =\left.\frac{R(u)}{(\log (y / u))^{\gamma}}\right|_{2} ^{\sqrt{y}}-\int_{2}^{\sqrt{y}} R(u) \frac{d}{d u}\left(\frac{1}{(\log (y / u))^{\gamma}}\right) d u \\
& =\frac{R(\sqrt{y})}{(\log \sqrt{y})^{\gamma}}-\frac{R(2)}{(\log (y / 2))^{\gamma}}-\int_{2}^{\sqrt{y}} \frac{\gamma R(u)}{u(\log (y / u))^{\gamma+1}} d u \\
& \ll_{\gamma} \frac{1 / \log y}{(\log y)^{\gamma}}+\frac{1}{(\log y)^{\gamma}}+\int_{2}^{\sqrt{y}} \frac{1 / \log u}{u(\log (y / u))^{\gamma+1}} d u \\
& \ll \gamma \frac{1}{(\log y)^{\gamma}}+\frac{1}{(\log y)^{\gamma+1}} \int_{2}^{\sqrt{y}} \frac{1}{u \log u} d u \\
& \ll \frac{1}{(\log y)^{\gamma}}+\frac{\log \log \sqrt{y}-\log \log 2}{(\log y)^{\gamma+1}}
\end{aligned}
$$

which, combined with equation (7), establishes the lemma.

Lemma 2.13. Let $\gamma>0$ such that $\gamma \notin \mathbb{N}$. For $y \geq 9$,

$$
\int_{\sqrt{y}}^{y / 3} \frac{1}{(u \log u)(\log (y / u))^{\gamma}} d u \ll_{\gamma} \frac{1}{(\log y)^{\min \{\gamma, 1\}}} .
$$

Proof. By Lemma 2.10,

$$
\begin{aligned}
\int_{\sqrt{y}}^{y / 3} \frac{1}{(u \log u)(\log (y / u))^{\gamma}} d u & =\left.\frac{H_{\gamma}\left(1-\frac{\log u}{\log y}\right)}{\gamma(\log (y / u))^{\gamma}}\right|_{\sqrt{y}} ^{y / 3} \\
& =\frac{H_{\gamma}\left(\frac{\log 3}{\log y}\right)}{\gamma(\log 3)^{\gamma}}-\frac{H_{\gamma}(1 / 2)}{\gamma\left(\frac{1}{2} \log y\right)^{\gamma}} \\
& \ll_{\gamma}\left|H_{\gamma}\left(\frac{\log 3}{\log y}\right)\right|+\frac{1}{(\log y)^{\gamma}} \ll_{\gamma} \frac{\log 3}{\log y}+\frac{1}{(\log y)^{\gamma}}
\end{aligned}
$$

by Lemma 2.8 (a); this bound is equivalent to the statement of the lemma. 
Lemma 2.14. Let $\gamma>0$ such that $\gamma \notin \mathbb{N}$, let $q$ be prime, and let $\alpha \in \mathbb{N}$. For $y \geq 9$,

$$
\sum_{\substack{y<p \leq y / 3 \\ \nu_{q}(p-1)=\alpha}} \frac{1}{p(\log (y / p))^{\gamma}} \ll_{\gamma} \frac{1}{(\log y)^{\min \{\gamma, 1\}}} .
$$

Proof. With $M(x)$ and $R(x)$ defined as in equations (5) and (6),

$$
\begin{aligned}
\sum_{\substack{y<p \leq y / 3 \\
\nu_{q}(p-1)=\alpha}} \frac{1}{p(\log (y / p))^{\gamma}} & =\int_{\sqrt{y}}^{y / 3} \frac{1}{(\log (y / u))^{\gamma}} d M(u) \\
& =\int_{\sqrt{y}}^{y / 3} \frac{1}{(\log (y / u))^{\gamma}} d\left(\frac{\log \log u}{q^{\alpha}}+c_{q^{\alpha}}-c_{q^{\alpha+1}}+R(u)\right) \\
& =\frac{1}{q^{\alpha}} \int_{\sqrt{y}}^{y / 3} \frac{1}{(\log (y / u))^{\gamma}} \frac{d u}{u \log u}+\int_{\sqrt{y}}^{y / 3} \frac{1}{(\log (y / u))^{\gamma}} d R(u) \\
& \ll_{\gamma} \frac{1}{(\log y)^{\min \{\gamma, 1\}}}+\int_{\sqrt{y}}^{y / 3} \frac{1}{(\log (y / u))^{\gamma}} d R(u)
\end{aligned}
$$

by Lemma 2.13, On the other hand, integrating by parts yields

$$
\begin{aligned}
\int_{\sqrt{y}}^{y / 3} \frac{1}{(\log (y / u))^{\gamma}} d R(u) & =\left.\frac{R(u)}{(\log (y / u))^{\gamma}}\right|_{\sqrt{y}} ^{y / 3}-\int_{\sqrt{y}}^{y / 3} R(u) \frac{d}{d u}\left(\frac{1}{(\log (y / u))^{\gamma}}\right) d u \\
& =\frac{R(y / 3)}{(\log 3)^{\gamma}}-\frac{R(\sqrt{y})}{(\log \sqrt{y})^{\gamma}}-\int_{\sqrt{y}}^{y / 3} \frac{\gamma R(u)}{u(\log (y / u))^{\gamma+1}} d u \\
& \ll_{\gamma} \frac{1}{\log y}+\frac{1 / \log y}{(\log y)^{\gamma}}+\int_{\sqrt{y}}^{y / 3} \frac{1 / \log u}{u(\log (y / u))^{\gamma+1}} d u \\
& \ll_{\gamma} \frac{1}{\log y}+\frac{1}{\log y} \int_{\sqrt{y}}^{y / 3} \frac{1}{(\log (y / u))^{\gamma+1}} \frac{d u}{u} .
\end{aligned}
$$

Using the change of variables $v=y / u$ yields

$$
\begin{aligned}
\int_{\sqrt{y}}^{y / 3} \frac{1}{(\log (y / u))^{\gamma}} d R(u) & \ll_{\gamma} \frac{1}{\log y}+\frac{1}{\log y} \int_{3}^{\sqrt{y}} \frac{1}{(\log v)^{\gamma+1}} \frac{d v}{v} \\
& =\frac{1}{\log y}+\left.\frac{1}{\log y} \frac{-\gamma}{(\log v)^{\gamma}}\right|_{3} ^{\sqrt{y}} \\
& \ll_{\gamma} \frac{1}{\log y}+\frac{1}{\log y}\left(1-\frac{1}{(\log y)^{\gamma}}\right) \ll \frac{1}{\log y}
\end{aligned}
$$

which, combined with equation (8), establishes the lemma.

Lemma 2.15. For $y \geq 9$,

$$
\sum_{\sqrt{y}<p \leq y / 3} \frac{1}{p \log (y / p)} \ll \frac{\log \log y}{\log y} .
$$


Proof. We first consider, for $2 \leq U \leq y^{3 / 4}$,

$$
\sum_{y / 2 U \leq p<y / U} \frac{1}{p \log (y / p)} \ll \frac{\pi(y / U)}{(y / U) \log U} \ll \frac{1}{\log (y / U) \log U} \ll \frac{1}{\log y \cdot \log U} .
$$

Applying this estimate with $U=2,4,8, \ldots$ until $U$ amply exceeds $\sqrt{y}$, we deduce that

$$
\sum_{y / 2 U \leq p<y / U} \frac{1}{p \log (y / p)} \leq \sum_{k=1}^{\log y} \sum_{y / 2^{k+1} \leq p<y / 2^{k}} \frac{1}{p \log (y / p)} \ll \sum_{k=1}^{\log y} \frac{1}{\log y \cdot \log 2^{k}} \ll \frac{\log \log y}{\log y} .
$$

Lemma 2.16. Let $\gamma>0$ such that $\gamma \notin \mathbb{N}$, let $q$ be prime, and let $\alpha \in \mathbb{N}$. For $y \geq 3$,

$$
\sum_{\substack{p \leq y / 3 \\ \nu_{q}(p-1)=\alpha}} \frac{1}{p(\log (y / p))^{\gamma}}=\frac{\log \log y}{q^{\alpha}(\log y)^{\gamma}}+O_{\gamma}\left(\frac{1}{(\log y)^{\min \{\gamma, 1\}}}\right) .
$$

Proof. Since the sum in question is empty when $3 \leq y<9$, we may assume that $y \geq 9$. By Lemmas 2.12 and 2.14,

$$
\begin{aligned}
\sum_{\substack{p \leq y / 3 \\
\nu_{q}(p-1)=\alpha}} \frac{1}{p(\log (y / p))^{\gamma}} & =\sum_{\substack{p \leq \sqrt{y} \\
\nu_{q}(p-1)=\alpha}} \frac{1}{p(\log (y / p))^{\gamma}}+\sum_{\substack{\sqrt{y}<p \leq y / 3 \\
\nu_{q}(p-1)=\alpha}} \frac{1}{p(\log (y / p))^{\gamma}} \\
& =\frac{\log \log y}{q^{\alpha}(\log y)^{\gamma}}+O_{\gamma}\left(\frac{1}{(\log y)^{\gamma}}\right)+O_{\gamma}\left(\frac{1}{(\log y)^{\min \{\gamma, 1\}}}\right)
\end{aligned}
$$

as desired.

Corollary 2.17. Let $0<\gamma \leq 1$, let $q$ be prime, and let $\alpha \in \mathbb{N}$. Then, uniformly for $y \geq 9$,

$$
\sum_{\substack{p \leq y / 3 \\ \nu_{q}(p-1)=\alpha}} \frac{1}{p(\log (y / p))^{\gamma}} \ll_{\gamma} \frac{\log \log y}{(\log y)^{\gamma}} .
$$

Proof. For $0<\gamma<1$ this estimate follows immediately from Lemma 2.16, while for $\gamma=1$ it follows from Lemmas 2.12 and 2.15.

We need only two more lemmas of this flavour before we begin to evaluate the inner sums in equation (2) in the next section.

Lemma 2.18. Let $k \geq 0$ and $\gamma>0$ be real numbers such that $\gamma \notin \mathbb{N}$, let $q$ be prime, and let $\alpha \in \mathbb{N}$. For $y \geq 9$,

$$
\sum_{\substack{p \leq y / 3 \\ \nu_{q}(p-1)=\alpha}} \frac{(\log \log (y / p))^{k}}{p(\log (y / p))^{\gamma}}=\frac{(\log \log y)^{k+1}}{q^{\alpha} \log ^{\gamma} y}+O_{k, \gamma}\left(\frac{(\log \log y)^{k}}{(\log y)^{\min \{\gamma, 1\}}}\right) .
$$

Proof. The upper part of the range of summation can be addressed by noting that

$$
\sum_{\substack{\sqrt{y}<p \leq y / 3 \\ \nu_{q}(p-1)=\alpha}} \frac{(\log \log (y / p))^{k}}{p(\log (y / p))^{\gamma}} \leq(\log \log y)^{k} \sum_{\substack{\sqrt{y}<p \leq y / 3 \\ \nu_{q}(p-1)=\alpha}} \frac{1}{p(\log (y / p))^{\gamma}} \ll_{\gamma} \frac{(\log \log y)^{k}}{(\log y)^{\min \{\gamma, 1\}}}
$$


by Lemma 2.14. As for the remainder of the range of summation, since $\log \log (y / p)=\log \log y+$ $O(1)$ for $p \leq \sqrt{y}$, we have

$$
\begin{aligned}
\sum_{\substack{p \leq \sqrt{y} \\
\nu_{q}(p-1)=\alpha}} \frac{(\log \log (y / p))^{k}}{p(\log (y / p))^{\gamma}} & =\left((\log \log y)^{k}+O_{k}\left((\log \log y)^{k-1}\right)\right) \sum_{\substack{p \leq \sqrt{y} \\
\nu_{q}(p-1)=\alpha}} \frac{1}{p(\log (y / p))^{\gamma}} \\
& =\left((\log \log y)^{k}+O_{k}\left((\log \log y)^{k-1}\right)\right)\left(\frac{\log \log y}{q^{\alpha}(\log y)^{\gamma}}+O_{\gamma}\left(\frac{1}{(\log y)^{\gamma}}\right)\right)
\end{aligned}
$$

by Lemma 2.12, Combining these two estimates establishes the lemma.

Lemma 2.19. Let $\gamma \in \mathbb{R}$, let $q$ be prime, and let $\alpha \in \mathbb{N}$. Then, uniformly for $y \geq 3$,

$$
\sum_{\substack{p \leq y / 3 \\ \nu_{q}(p-1)=\alpha}} \frac{1}{p^{2}(\log (y / p))^{\gamma}} \ll_{\gamma}(\log y)^{-\gamma} .
$$

Proof. Indeed, the desired bound holds even if we ignore the condition $\nu_{q}(p-1)=\alpha$, since

$$
\begin{aligned}
\sum_{\substack{p \leq y / 3 \\
\nu_{q}(p-1)=\alpha}} \frac{1}{p^{2}(\log (y / p))^{\gamma}} & \leq \sum_{p \leq \sqrt{y}} \frac{1}{p^{2}(\log (y / p))^{\gamma}}+\sum_{p \leq \sqrt{y}} \frac{1}{p^{2}(\log (y / p))^{\gamma}} \\
& \leq \sum_{p \leq \sqrt{y}} \frac{1}{p^{2}(\log \sqrt{y})^{\gamma}}+\sum_{n>\sqrt{y}} \frac{1}{n^{2}(\log 3)^{\gamma}} \\
& \ll_{\gamma}(\log y)^{-\gamma} \sum_{p} \frac{1}{p^{2}}+\frac{1}{\sqrt{y}} \ll(\log y)^{-\gamma}
\end{aligned}
$$

2.4. Recursive evaluation of iterated sums. The technical lemmas in the previous section hint at the types of expressions that will appear as we work our way through the nested sums in equation (2). In this section we establish the results that allow us to recursively evaluate these expressions asymptotically.

Proposition 2.20. Let $k \geq 0$ and $\gamma>0$ be real numbers such that $\gamma \notin \mathbb{N}$, let $\left\{w_{1}, w_{2}, \ldots, w_{n}\right\}$ be a set of $n$ distinct primes, let $q$ be prime, and let $\alpha \in \mathbb{N}$. For $y \geq 3$,

$$
\begin{aligned}
& \sum_{\substack{p \leq y / 3 \\
p \neq w_{1}, w_{2}, \ldots, w_{n} \\
\nu_{q}(p-1)=\alpha}} \frac{p+O(1)}{p^{2}}\left(\frac{(\log \log (y / p))^{k}}{(\log (y / p))^{\gamma}}+O\left(\frac{(\log \log y)^{k-1}}{(\log (y / p))^{\min \{\gamma, 1\}}}\right)\right) \\
& \quad=\frac{(\log \log y)^{k+1}}{q^{\alpha} \log ^{\gamma} y}+O_{n, k, \gamma}\left(\frac{(\log \log y)^{k}}{(\log y)^{\min \{\gamma, 1\}}}\right) .
\end{aligned}
$$


Proof. Since the sum in question is empty when $3 \leq y<9$, we may assume that $y \geq 9$. We begin by writing

$$
\begin{aligned}
\sum_{\substack{p \leq y / 3 \\
p \neq w_{1}, w_{2}, \ldots, w_{n} \\
\nu_{q}(p-1)=\alpha}} \frac{p+O(1)}{p^{2}}\left(\frac{(\log \log (y / p))^{k}}{(\log (y / p))^{\gamma}}+O\left(\frac{(\log \log y)^{k-1}}{(\log (y / p))^{\min \{\gamma, 1\}}}\right)\right) \\
=\left\{\sum_{\substack{p \leq y / 3 \\
\nu_{q}(p-1)=\alpha}} \frac{(\log \log (y / p))^{k}}{p(\log (y / p))^{\gamma}}+O\left(\sum_{\substack{1 \leq i \leq n \\
w_{i} \leq y / 3}} \frac{\left(\log \log \left(y / w_{i}\right)\right)^{k}}{w_{i}\left(\log \left(y / w_{i}\right)\right)^{\gamma}}\right)\right\} \\
\quad+O\left(\sum_{\substack{p \leq y / 3 \\
\nu_{q}(p-1)=\alpha}} \frac{(\log \log (y / p))^{k}}{p^{2}(\log (y / p))^{\gamma}}+\sum_{\substack{p \leq y / 3 \\
\nu_{q}(p-1)=\alpha}} \frac{(\log \log y)^{k-1}}{\left.p(\log (y / p))^{\min \{\gamma, 1\}}\right)}\right. \\
=\frac{(\log \log y)^{k+1}}{q^{\alpha} \log { }^{\gamma} y}+O_{k, \gamma}\left(\frac{(\log \log y)^{k}}{(\log y)^{\min \{\gamma, 1\}}}\right)+O\left(\sum_{\substack{1 \leq i \leq n \\
w_{i} \leq y / 3}} \frac{(\log \log y)^{k}}{w_{i}\left(\log \left(y / w_{i}\right)\right)^{\gamma}}\right) \\
\quad+\sum_{\substack{p \leq y / 3 \\
\nu_{q}(p-1)=\alpha}} \frac{(\log \log y)^{k-1}}{p^{2}(\log (y / p))^{\gamma}}+\sum_{\substack{p \leq y / 3 \\
\nu_{q}(p-1)=\alpha}} \frac{\left.(\log (y / p))^{\min \{\gamma, 1\}}\right)}{p(\log y)^{k}}
\end{aligned}
$$

by Lemma 2.18. Since $w_{i} \log \left(y / w_{i}\right) \gg \log y$ for $y \geq 9$ and $w_{i} \leq y / 3$, the first error term sum on the right-hand side is $\ll_{n}(\log \log y)^{k}(\log y)^{-\gamma}$, while

$$
\begin{gathered}
\sum_{\substack{p \leq y / 3 \\
\nu_{q}(p-1)=\alpha}} \frac{(\log \log y)^{k}}{p^{2}(\log (y / p))^{\gamma}} \ll_{\gamma} \frac{(\log \log y)^{k}}{(\log y)^{\gamma}} \\
\sum_{\substack{p \leq y / 3 \\
\nu_{q}(p-1)=\alpha}} \frac{(\log \log y)^{k-1}}{p(\log (y / p))^{\min \{\gamma, 1\}}} \ll_{\gamma}(\log \log y)^{k-1} \frac{\log \log y}{(\log y)^{\min \{\gamma, 1\}}}
\end{gathered}
$$

by Lemma 2.19 and Corollary 2.17. Therefore the error terms in equation (10) are all majorized by the error term in equation (9).

Definition 2.21. Let $k$ be a nonnegative real number, and let $q$ be prime. Define

$$
S_{q}(x ; k)=\frac{(\log \log x)^{k}}{(\log x)^{1 /(q-1)}}+O_{q}\left(\frac{(\log \log x)^{k-1}}{(\log x)^{1 /(q-1)}}\right) .
$$

Further, for $\alpha_{1}, \ldots, \alpha_{i} \in \mathbb{N}$, define

$$
\begin{aligned}
S_{q}\left(x ; k ; \alpha_{1}, \ldots, \alpha_{i}\right) & \sum_{\begin{array}{c}
p_{1} \leq x / 3 \\
\nu_{q}\left(p_{1}-1\right)=\alpha_{1}
\end{array}} \frac{p_{1}+O(1)}{p_{1}^{2}} \sum_{\begin{array}{c}
p_{2} \leq x / 3 p_{1} \\
p_{2} \neq p_{1} \\
\nu_{q}\left(p_{2}-1\right)=\alpha_{2}
\end{array}} \frac{p_{2}+O(1)}{p_{2}^{2}} \cdots \sum_{\substack{p_{i} \leq x / 3 p_{1} \cdots p_{i-1} \\
p_{i} \neq p_{1}, \ldots, p_{i-1} \\
\nu_{q}\left(p_{i}-1\right)=\alpha_{i}}}\left\{\frac{p_{i}+O(1)}{p_{i}^{2}}\right. \\
& \left.\times\left(\frac{\left(\log \log \left(x / p_{1} \cdots p_{i}\right)\right)^{k}}{\left(\log \left(x / p_{1} \cdots p_{i}\right)\right)^{1 /(q-1)}}+O_{q}\left(\frac{\left(\log \log \left(x / p_{1} \cdots p_{i}\right)\right)^{k-1}}{\left(\log \left(x / p_{1} \cdots p_{i}\right)\right)^{1 /(q-1)}}\right)\right)\right\} .
\end{aligned}
$$


Note that the expressions $S_{q}(x ; k)$ and $S_{q}\left(x ; k ; \alpha_{1}, \ldots, \alpha_{i}\right)$ are given by asymptotic, not explicit, formulas. For instance, when $i=1$, applying Proposition 2.20 yields

$$
\begin{aligned}
S_{q}\left(x ; k ; \alpha_{1}\right) & =\sum_{\substack{p_{1} \leq x / 3 \\
\nu_{q}\left(p_{1}-1\right)=\alpha_{1}}} \frac{p_{1}+O(1)}{p_{1}^{2}}\left(\frac{\left(\log \log \left(x / p_{1}\right)\right)^{k}}{\left(\log \left(x / p_{1}\right)\right)^{1 /(q-1)}}+O_{q}\left(\frac{\left(\log \log \left(x / p_{1}\right)\right)^{k-1}}{\left(\log \left(x / p_{1}\right)\right)^{1 /(q-1)}}\right)\right) \\
& =\frac{(\log \log x)^{k+1}}{q^{\alpha_{1}}(\log x)^{1 /(q-1)}}+O_{q}\left(\frac{(\log \log x)^{k}}{(\log x)^{1 /(q-1)}}\right),
\end{aligned}
$$

which, by comparison to equation (11), is the same as the expression $S_{q}(x ; k+1) / q^{\alpha_{1}}$. Here, we are not claiming that $S_{q}\left(x ; k ; \alpha_{1}\right)$ must be exactly equal to $S_{q}(x ; k+1) / q^{\alpha_{1}}$, but rather that these two expressions have identical main terms and error terms of equal magnitude; in particular, we may freely replace $S_{q}\left(x ; k ; \alpha_{1}\right)$ by $S_{q}(x ; k+1) / q^{\alpha_{1}}$ in any expression.

This observation generalizes to any natural number $i$, resulting in the following proposition.

Proposition 2.22. Let $k$ be a nonnegative real number, let $q$ be prime, and let $\alpha_{1}, \ldots, \alpha_{j} \in \mathbb{N}$. For any $1 \leq i \leq j$, the expressions

$$
S_{q}\left(x ; k ; \alpha_{1}, \ldots, \alpha_{i}\right) \quad \text { and } \quad q^{-\alpha_{i}} S_{q}\left(x ; k+1 ; \alpha_{1}, \ldots, \alpha_{i-1}\right)
$$

have the same main terms and error terms of equal magnitude, so that we may freely replace $S_{q}\left(x ; k ; \alpha_{1}, \ldots, \alpha_{i}\right)$ with $q^{-\alpha_{i}} S_{q}\left(x ; k+1 ; \alpha_{1}, \ldots, \alpha_{i-1}\right)$ in any expression. In particular, the expressions

$$
S_{q}\left(x ; k ; \alpha_{1}, \ldots, \alpha_{j}\right) \quad \text { and } q^{-\sum_{i=1}^{j} \alpha_{i}} S_{q}(x ; k+j)
$$

have the same main terms and error terms of equal magnitude, so that we may freely replace $S_{q}\left(x ; k ; \alpha_{1}, \ldots, \alpha_{j}\right)$ with $q^{-\sum_{i=1}^{j} \alpha_{i}} S_{q}(x ; k+j)$ in any expression (as long as we note that the error term in equation (11) will depend on $j$ as well as $q$ ).

Proof. Applying Proposition 2.20, with $y=x / p_{1} \ldots p_{i-1}$, to the innermost sum in Definition 2.21, we see that

$$
\begin{aligned}
S_{q}\left(x ; k ; \alpha_{1}, \ldots, \alpha_{i}\right) & \\
= & \sum_{\substack{p_{1} \leq x / 3 \\
\nu_{q}\left(p_{1}-1\right)=\alpha_{1}}} \frac{p_{1}+O(1)}{p_{1}^{2}} \cdots \sum_{\begin{array}{c}
p_{i-1} \leq x / 3 p_{1} \cdots p_{i-2} \\
p_{i-1 \neq p_{1}, \ldots, p_{i-2}} \\
\nu_{q}\left(p_{i-1}-1\right)=\alpha_{i-1}
\end{array}}\left\{\frac{p_{1}+O(1)}{p_{1}^{2}}\right. \\
& \left.\times\left(\frac{\left(\log \log \left(x / p_{1} \cdots p_{i-1}\right)\right)^{k+1}}{q^{\alpha_{i}}\left(\log \left(x / p_{1} \cdots p_{i-1}\right)\right)^{1 /(q-1)}}+O_{q}\left(\frac{\left(\log \log \left(x / p_{1} \cdots p_{i-1}\right)\right)^{k}}{\left(\log \left(x / p_{1} \cdots p_{i-1}\right)\right)^{1 /(q-1)}}\right)\right)\right\} .
\end{aligned}
$$

The last assertion follows from a trivial induction.

Definition 2.23. Let $\gamma$ be a positive real number, and let $q$ be prime. Define $\varepsilon_{q}(x, \gamma)=(\log x)^{-\gamma}$. Further, for any $\alpha_{1}, \ldots, \alpha_{i} \in \mathbb{N}$, define

$$
\varepsilon_{q}\left(x, \gamma ; \alpha_{1}, \ldots, \alpha_{i}\right)=\sum_{\substack{p_{1} \leq x / 3 \\ \nu_{q}\left(p_{1}-1\right)=\alpha_{1}}} \frac{1}{p_{1}} \sum_{\substack{p_{2} \leq x / 3 p_{1} \\ p_{2} \neq p_{1} \\ \nu_{q}\left(p_{2}-1\right)=\alpha_{2}}} \frac{1}{p_{2}} \cdots \sum_{\substack{p_{i} \leq x / 3 p_{1} \cdots p_{i-1} \\ p_{i} \neq p_{1}, \ldots, p_{i-1} \\ \nu_{q}\left(p_{i}-1\right)=\alpha_{i}}} \frac{1}{p_{i}}\left(\log \frac{x}{p_{1} \cdots p_{i}}\right)^{-\gamma} .
$$


Proposition 2.24. Let $\gamma$ be a positive real number, let $q$ be prime, and let $\alpha_{1}, \ldots, \alpha_{j} \in \mathbb{N}$. For any $1 \leq i \leq j$ and for any $x \geq 3$,

$$
\varepsilon_{q}\left(x, \gamma ; \alpha_{1}, \ldots, \alpha_{i}\right) \ll_{q} \varepsilon_{q}\left(x, \gamma ; \alpha_{1}, \ldots, \alpha_{i-1}\right) \log \log x .
$$

In particular, $\varepsilon_{q}\left(x, \gamma ; \alpha_{1}, \ldots, \alpha_{j}\right) \ll_{q, j}(\log \log x)^{j} /(\log x)^{\gamma}$.

Proof. If $x / p_{1} \ldots p_{i-1}<3$ then the innermost sum in the definition of $\varepsilon_{q}\left(x, \gamma ; \alpha_{1}, \ldots, \alpha_{i}\right)$ is empty; otherwise, applying Lemma 2.16 (with $y=x / p_{1} \ldots p_{i-1}$ ) to the innermost sum, we obtain

$$
\begin{aligned}
& \varepsilon_{q}\left(x, \gamma ; \alpha_{1}, \ldots, \alpha_{i}\right) \\
& \ll \sum_{\substack{p_{1} \leq x / 3 \\
\nu_{q}\left(p_{1}-1\right)=\alpha_{1}}} \frac{1}{p_{1}} \sum_{\substack{p_{2} \leq x / 3 p_{1} \\
p_{2} \neq p_{1} \\
\nu_{q}\left(p_{2}-1\right)=\alpha_{2}}} \frac{1}{p_{2}} \cdots \sum_{\begin{array}{c}
p_{i-1} \leq x / 3 p_{1} \cdots p_{i-2} \\
p_{i}-1 \neq p_{1}, \ldots, p_{i-2} \\
\nu_{q}\left(p_{i-1}-1\right)=\alpha_{i-1}
\end{array}} \frac{1}{p_{i-1}}\left(\frac{\log \log \left(x / p_{1} \cdots p_{i-1}\right)}{q^{\alpha_{i}}\left(\log \left(x / p_{1} \cdots p_{i-1}\right)\right)^{\gamma}}\right) \\
& \ll \sum_{\substack{p_{1} \leq x / 3 \\
\nu_{q}\left(p_{1}-1\right)=\alpha_{1}}} \frac{1}{p_{1}} \sum_{\substack{p_{2} \leq x / 3 p_{1} \\
p_{2} \neq p_{1} \\
\nu_{q}\left(p_{2}-1\right)=\alpha_{2}}} \frac{1}{p_{2}} \cdots \sum_{\substack{p_{i-1} \leq x / 3 p_{1} \cdots p_{i-2} \\
p_{i-1} \neq p_{1}, \ldots, p_{i-2} \\
\nu_{q}\left(p_{i-1}-1\right)=\alpha_{i-1}}} \frac{1}{p_{i-1}}\left(\frac{\log \log x}{\left(\log \left(x / p_{1} \cdots p_{i-1}\right)\right)^{\gamma}}\right) \\
& =\varepsilon_{q}\left(x, \gamma ; \alpha_{1}, \ldots, \alpha_{i-1}\right) \log \log x .
\end{aligned}
$$

(We check that this calculation is valid even in the case $i=1$, where the above notation is obfuscatory.) The last assertion follows from the definition of $\varepsilon_{q}(x, \gamma)$ and a trivial induction.

2.5. Evaluation of $D_{0}(H, x)$. We now have all the tools we need to evaluate the counting function $D_{0}(H, x)$ from Definition 2.2, which is the majority of the work needed to establish Theorem 1.4 . Since we can apply Proposition 2.6 only when $x / p_{1} \cdots p_{j} \geq 3$, we start by splitting the sum in equation (2), so that Lemma 2.4 becomes

$$
\begin{aligned}
& D_{0}\left(\mathbb{Z}_{q} \boldsymbol{\alpha}, x\right)=C(\boldsymbol{\alpha})\left(\sum_{\substack{p_{1} \leq x / 3 \\
\nu_{q}\left(p_{1}-1\right)=\alpha_{1}}} \sum_{\substack{p_{2} \leq x / 3 p_{1} \\
p_{2} \neq p_{1} \\
\nu_{q}\left(p_{2}-1\right)=\alpha_{2}}} \cdots \sum_{\begin{array}{c}
p_{j} \leq x / 3 p_{1} \cdots p_{j-1} \\
p_{j} \neq p_{1}, \ldots, p_{j}-1 \\
\nu_{q}\left(p_{j}-1\right)=\alpha_{j}
\end{array} \quad\left(t \mid m \text { and } t \equiv 1(\bmod q) \Rightarrow t \in\left\{p_{1}, \ldots, p_{j}\right\}\right.} \sum_{\substack{m \leq x / p_{1} \cdots p_{j} \\
q \nmid m}} 1+\right.
\end{aligned}
$$

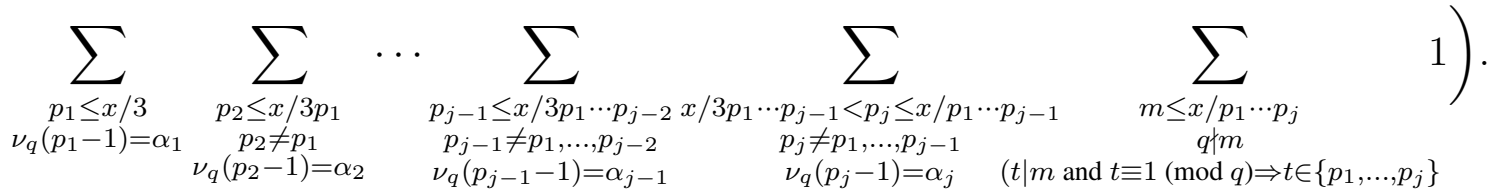

In the next two propositions we estimate the second sum in equation (12) and then asymptotically evaluate the first sum.

Lemma 2.25. Let $q$ be an odd prime, and let $\boldsymbol{\alpha}=\left(\alpha_{1}, \ldots, \alpha_{j}\right)$ be a partition. For $x \geq 3$,

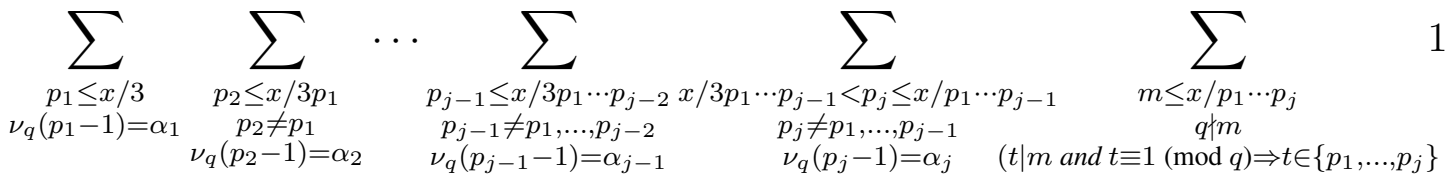

$$
\begin{aligned}
& \ll_{q, j} \frac{x(\log \log x)^{j-1}}{\log x} .
\end{aligned}
$$


Proof. Since $x / p_{1} p_{2} \cdots p_{j}<3$, the innermost sum has at most two terms, and thus

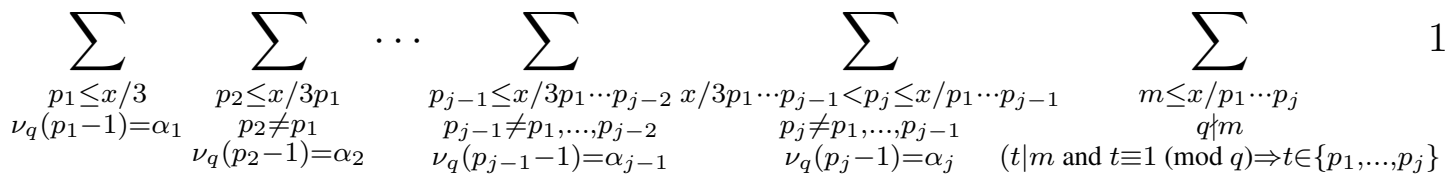

$$
\begin{aligned}
& \ll \sum_{\substack{p_{1} \leq x / 3 \\
\nu_{q}\left(p_{1}-1\right)=\alpha_{1}}} \sum_{\substack{p_{2} \leq x / 3 p_{1} \\
p_{2} \neq p_{1} \\
\nu_{q}\left(p_{2}-1\right)=\alpha_{2}}} \cdots \sum_{\substack{p_{j-1} \leq x / 3 p_{1} \cdots p_{j-2} \\
p_{j-1} \neq p_{1}, \ldots, p_{j-2} \\
\nu_{q}\left(p_{j-1}-1\right)=\alpha_{j-1}}} \sum_{\substack{x p_{1} \cdots p_{j-1}<p_{j} \leq x / p_{1} \cdots p_{j-1} \\
p_{j} \neq p_{1}, \ldots, p_{j-1} \\
\nu_{q}\left(p_{j}-1\right)=\alpha_{j}}} 1 .
\end{aligned}
$$

If $x / p_{1} \cdots p_{j-1}<3$ then the innermost sum vanishes; otherwise Chebyshev's estimate gives

$$
\begin{aligned}
& \sum_{\substack{p_{1} \leq x / 3 \\
\nu_{q}\left(p_{1}-1\right)=\alpha_{1}}} \sum_{\substack{p_{2} \leq x / 3 p_{1} \\
p_{2} \neq p_{1} \\
\nu_{q}\left(p_{2}-1\right)=\alpha_{2}}} \cdots \sum_{\begin{array}{c}
p_{j-1} \leq x / 3 p_{1} \cdots p_{j-2} \\
p_{j-1} \neq p_{1}, \ldots, p_{j-2} \\
\nu_{q}\left(p_{j-1}-1\right)=\alpha_{j-1}
\end{array}} \sum_{\substack{x / 3 p_{1} \cdots p_{j-1}<p_{j} \leq x / p_{1} \cdots p_{j-1} \\
p_{j} \neq p_{1}, \ldots, p_{j-1} \\
\nu_{q}\left(p_{j}-1\right)=\alpha_{j}}} 1 \\
& \leq \sum_{\substack{p_{1} \leq x / 3 \\
\nu_{q}\left(p_{1}-1\right)=\alpha_{1}}} \sum_{\substack{p_{2} \leq x / 3 p_{1} \\
p_{2} \neq p_{1} \\
\nu_{q}\left(p_{2}-1\right)=\alpha_{2}}} \cdots \sum_{\substack{p_{j-1} \leq x / 3 p_{1} \cdots p_{j-2} \\
p_{j-1} \neq p_{1}, \ldots, p_{j-2} \\
\nu_{q}\left(p_{j-1}-1\right)=\alpha_{j-1}}} \pi\left(x / p_{1} p_{2} \cdots p_{j-1}\right) \\
& \begin{array}{l}
\ll \sum_{\substack{p_{1} \leq x / 3 \\
\nu_{q}\left(p_{1}-1\right)=\alpha_{1}}} \sum_{\substack{p_{2} \leq x / 3 p_{1} \\
p_{2} \neq p_{1} \\
\nu_{q}\left(p_{2}-1\right)=\alpha_{2}}} \cdots \sum_{\substack{p_{j-1} \leq x / 3 p_{1} \cdots p_{j-2} \\
p_{j-1} \neq p_{1}, \ldots, p_{j-2} \\
\nu_{q}\left(p_{j-1}-1\right)=\alpha_{j-1}}} \frac{x / p_{1} \cdots p_{j-1}}{\log \left(x / p_{1} \cdots p_{j-1}\right)} \\
=x \varepsilon_{q}\left(x, 1 ; \alpha_{1}, \ldots, \alpha_{j-1}\right)
\end{array}
\end{aligned}
$$

in the notation of Definition 2.23. The lemma now follows directly from Proposition 2.24,

Proposition 2.26. Let $q$ be an odd prime, and let $\alpha_{1}, \ldots, \alpha_{j} \in \mathbb{N}$. For any $x \geq 3$,

$$
\begin{gathered}
\sum_{\substack{p_{1} \leq x / 3 \\
\nu_{q}\left(p_{1}-1\right)=\alpha_{1}}} \sum_{\substack{p_{2} \leq x / 3 p_{1} \\
p_{2} \neq p_{1} \\
\nu_{q}\left(p_{2}-1\right)=\alpha_{2}}} \cdots \sum_{\substack{p_{j} \leq x / 3 p_{1} \cdots p_{j-1} \\
p_{j} \neq p_{1}, \ldots, p_{j-1} \\
\nu_{q}\left(p_{j}-1\right)=\alpha_{j}}} \sum_{\substack{m \leq x / p_{1} \cdots p_{j} \\
q \nmid m \\
\left(t \mid m \text { and } t \equiv 1(\bmod q) \Rightarrow t \in\left\{p_{1}, \ldots, p_{j}\right\}\right.}} 1 \\
=\frac{B_{q}}{q^{\sum_{i=1}^{j} \alpha_{i}}} \frac{x(\log \log x)^{j}}{(\log x)^{1 /(q-1)}}+O_{q}\left(\frac{x(\log \log x)^{j-1}}{(\log x)^{1 /(q-1)}}\right)
\end{gathered}
$$

where $B_{q}$ is as in Definition 2.5

Proof. Throughout this proof, let $J$ denote the left-hand side of equation (13). Note that the condition $p_{j} \leq x / 3 p_{1} \cdots p_{j-1}$ in the second-to-last sum implies that the bound $x / p_{1} \cdots p_{j}$ in the 
innermost sum is at least 3. Therefore, by Proposition 2.6.

$$
\begin{aligned}
& J=\sum_{\substack{p_{1} \leq x / 3 \\
\nu_{q}\left(p_{1}-1\right)=\alpha_{1}}} \sum_{\substack{p_{2} \leq x / 3 p_{1} \\
p_{2} \neq p_{1} \\
\nu_{q}\left(p_{2}-1\right)=\alpha_{2}}} \cdots \sum_{\substack{p_{j} \leq x / 3 p_{1} \cdots p_{j-1} \\
p_{j} \neq p_{1}, \cdots, p_{j-1} \\
\nu_{q}\left(p_{j}-1\right)=\alpha_{j}}}\left\{B_{q} \frac{x}{p_{1} \cdots p_{j}}\left(\log \frac{x}{p_{1} \cdots p_{j}}\right)^{-1 /(q-1)} \prod_{i=1}^{j}\left(1-\frac{1}{p_{i}}\right)^{-1}\right. \\
& \left.+O\left(\frac{x}{p_{1} \cdots p_{j}}\left(\log \frac{x}{p_{1} \cdots p_{j}}\right)^{-1-1 /(q-1)}\right)\right\} \\
& =B_{q} x \sum_{\substack{p_{1} \leq x / 3 \\
\nu_{q}\left(p_{1}-1\right)=\alpha_{1}}} \cdots \sum_{\substack{p_{j} \leq x / 3 p_{1} \cdots p_{j-1} \\
p_{j} \neq p_{1}, \cdots, p_{j-1} \\
\nu_{q}\left(p_{j}-1\right)=\alpha_{j}}} \frac{1}{p_{1} \cdots p_{j}}\left(\log \frac{x}{p_{1} \cdots p_{j}}\right)^{-1 /(q-1)} \prod_{i=1}^{j}\left(1-\frac{1}{p_{i}}\right)^{-1} \\
& +O\left(x \sum_{\substack{p_{1} \leq x / 3 \\
\nu_{q}\left(p_{1}-1\right)=\alpha_{1}}} \sum_{\substack{p_{2} \leq x / 3 p_{1} \\
p_{2} \neq p_{1} \\
\nu_{q}\left(p_{2}-1\right)=\alpha_{2}}} \cdots \sum_{\substack{p_{j} \leq x / 3 p_{1} \cdots p_{j-1} \\
p_{j} \neq p_{1}, \ldots, p_{j-1} \\
\nu_{q}\left(p_{j}-1\right)=\alpha_{j}}} \frac{1}{p_{1} \cdots p_{j}}\left(\log \frac{x}{p_{1} \cdots p_{j}}\right)^{-1-1 /(q-1)}\right) \\
& =B_{q} x S_{q}\left(x, 0 ; \alpha_{1}, \ldots, \alpha_{j}\right)+O\left(x \varepsilon_{q}\left(x, 1+\frac{1}{q-1} ; \alpha_{1}, \ldots, \alpha_{j}\right)\right)
\end{aligned}
$$

in the notation of Definitions 2.21 and 2.23. Thus, by Propositions 2.22 and 2.24,

$$
\begin{aligned}
J & =B_{q} x \frac{1}{q^{\sum_{i=1}^{j} \alpha_{i}}} S_{q}(x, j)+O\left(\frac{x(\log \log x)^{j}}{(\log x)^{1+1 /(q-1)}}\right) \\
& =B_{q} x \frac{1}{q^{\sum_{i=1}^{j} \alpha_{i}}}\left(\frac{(\log \log x)^{j}}{(\log x)^{1 /(q-1)}}+O_{q}\left(\frac{(\log \log x)^{j-1}}{(\log x)^{1 /(q-1)}}\right)\right)+O\left(\frac{x(\log \log x)^{j}}{(\log x)^{1+1 /(q-1)}}\right)
\end{aligned}
$$

by equation (11), which establishes the proposition.

The work in this section leads immediately to an asymptotic formula for $D_{0}\left(\mathbb{Z}_{q^{\alpha}}, x\right)$.

Theorem 2.27. Let $q$ be an odd prime, and let $\boldsymbol{\alpha}=\left(\alpha_{1}, \ldots, \alpha_{j}\right)$ be a partition. For any $x \geq 3$,

$$
D_{0}\left(\mathbb{Z}_{q^{\boldsymbol{\alpha}}}, x\right)=C(\boldsymbol{\alpha}) \frac{B_{q}}{q^{\sum_{i=1}^{j} \alpha_{i}}} \frac{x(\log \log x)^{j}}{(\log x)^{1 /(q-1)}}+O_{q, \boldsymbol{\alpha}}\left(\frac{x(\log \log x)^{j-1}}{(\log x)^{1 /(q-1)}}\right) .
$$

Proof. Thanks to the expression (12), the theorem follows immediately from Proposition 2.26 and Lemma 2.25.

2.6. Proof of Theorem 1.4. As mentioned earlier, each of the counting functions $D_{k}(H, x)$, if nonzero, is equal to $D_{0}\left(\mathbb{Z}_{H^{\prime}}, x\right)$ for a certain $q$-group $H^{\prime}$ depending on $H$ and $k$. We make this precise enough for our purposes in the following lemma.

Lemma 2.28. Let $q$ be an odd prime, let $\boldsymbol{\alpha}=\left(\alpha_{1}, \ldots, \alpha_{j}\right)$ be a partition, and let $k \in \mathbb{N}$.

(a) When $k=1$, we have $D_{1}\left(\mathbb{Z}_{q^{\alpha}}, x\right)=D_{0}\left(\mathbb{Z}_{q^{\alpha}}, \frac{x}{q}\right)$.

(b) If $k \geq \alpha_{1}+2$, then $D_{k}\left(\mathbb{Z}_{q^{\alpha}}, x\right)=0$.

(c) For any $k \geq 2$, we have $D_{k}\left(\mathbb{Z}_{q^{\alpha}}, x\right) \ll_{q, \boldsymbol{\alpha}, k} x(\log \log x)^{j-1} /(\log x)^{1 /(q-1)}$. 
Proof. In all parts, the integers counted by $D_{k}\left(\mathbb{Z}_{q^{\alpha}}, x\right)=\#\left\{n \leq x: \nu_{q}(n)=k, G_{q}(n)=\mathbb{Z}_{q^{\alpha}}\right\}$ can be written as $n=q^{k} m$ where $m \leq \frac{x}{q^{k}}$ and $q \nmid m$; we also have $\mathbb{Z}_{n}^{\times} \cong \mathbb{Z}_{q^{k-1}(q-1)} \times \mathbb{Z}_{m}^{\times}$, and in particular the Sylow $q$-subgroup of $\mathbb{Z}_{n}^{\times}$is congruent to the product of $\mathbb{Z}_{q^{k-1}}$ and the Sylow $q$-subgroup of $\mathbb{Z}_{m}^{\times}$.

When $k=1$, these two Sylow $q$-subgroups are identical, and therefore $D_{1}\left(\mathbb{Z}_{q^{\alpha}}, x\right)=D_{0}\left(\mathbb{Z}_{q^{\alpha}}, \frac{x}{q}\right)$ as claimed in part (a).

When $k \geq \alpha_{1}+2$, the Sylow $q$-subgroup of $\mathbb{Z}_{n}^{\times}$will include a copy of $\mathbb{Z}_{q^{k-1}}$; since the largest primary subgroup of $\mathbb{Z}_{q^{\alpha}}$ is $\mathbb{Z}_{q^{\alpha_{1}}}$, the fact that $k-1>\alpha_{1}$ makes it impossible for that Sylow $q$-subgroup to equal $\mathbb{Z}_{q^{\alpha}}$, as claimed in part (b). The same is true if $k-1 \leq \alpha_{1}$ but $k-1 \notin \boldsymbol{\alpha}$.

Finally, suppose $k \geq 2$ and $k-1 \in \boldsymbol{\alpha}$. Let $\boldsymbol{\alpha}^{\prime}=\boldsymbol{\alpha} \backslash\{k-1\}$ denote the partition obtained from $\boldsymbol{\alpha}$ by removing one occurrence of $k-1$. Then the fact that $\mathbb{Z}_{n}^{\times} \cong \mathbb{Z}_{q^{k-1}(q-1)} \times \mathbb{Z}_{m}^{\times}$implies that the Sylow $q$-subgroup of $\mathbb{Z}_{m}^{\times}$equals $\mathbb{Z}_{q^{\alpha^{\prime}}}$, and therefore

$$
D_{k}\left(\mathbb{Z}_{q^{\boldsymbol{\alpha}}}, x\right)=D_{0}\left(\mathbb{Z}_{q^{\boldsymbol{\alpha}^{\prime}}}, \frac{x}{q^{k}}\right) \ll_{q, \boldsymbol{\alpha}^{\prime}} \frac{\left(x / q^{k}\right)\left(\log \log \left(x / q^{k}\right)\right)^{j-1}}{\left(\log \left(x / q^{k}\right)\right)^{1 /(q-1)}} \ll_{q, \boldsymbol{\alpha}, k} \frac{x(\log \log x)^{j-1}}{(\log x)^{1 /(q-1)}}
$$

as claimed in part (c).

We have now completed the last preparatory step necessary to prove our main theorem, which we do after defining the leading constant that emerges from the calculation.

Definition 2.29. For a prime $q$ and a partition $\boldsymbol{\alpha}=\left(\alpha_{1}, \ldots, \alpha_{j}\right)$, define

$$
E_{q}(\boldsymbol{\alpha})=\frac{q+1}{q^{1+\sum_{i=1}^{j} \alpha_{i}}} .
$$

Recall also $B_{q}$ and $C(\boldsymbol{\alpha})$ from Definition 2.5 and equation (1), respectively:

$$
\begin{aligned}
& B_{q}=\frac{1}{\Gamma(1-1 /(q-1))}\left(1-\frac{1}{q}\right)^{1-1 /(q-1)} \prod_{\substack{p \neq q \\
p \neq 1(\bmod q)}}\left(1-\frac{1}{p^{k_{p}}}\right)^{-1 / k_{p}} \prod_{\chi \neq \chi_{0}} L(1, \chi)^{-1 /(q-1)} \\
& C(\boldsymbol{\alpha})=\prod_{u=1}^{\infty} \frac{1}{\left(a_{u}-a_{u+1}\right) !}=\prod_{u=1}^{\alpha_{1}} \frac{1}{\left(a_{u}-a_{u+1}\right) !}
\end{aligned}
$$

where $\left(a_{1}, a_{2}, \ldots\right)$ is the conjugate of the partition $\boldsymbol{\alpha}$. Then, given the finite abelian $q$-group $\mathbb{Z}_{q^{\alpha}}$, define the constant

$$
K\left(\mathbb{Z}_{q^{\alpha}}\right)=B_{q} C(\boldsymbol{\alpha}) E_{q}(\boldsymbol{\alpha}) .
$$

Proof of Theorem 1.4 Write $\boldsymbol{\alpha}=\left(\alpha_{1}, \ldots, \alpha_{j}\right)$. Beginning with Definition 2.2,

$$
\begin{aligned}
D\left(\mathbb{Z}_{q^{\alpha}}, x\right)=\sum_{k=0}^{\infty} D_{k}\left(\mathbb{Z}_{q^{\alpha}}, x\right) & =\sum_{k=0}^{\alpha_{1}+1} D_{k}\left(\mathbb{Z}_{q^{\alpha}}, x\right) \\
& =D_{0}\left(\mathbb{Z}_{q^{\alpha}}, x\right)+D_{0}\left(\mathbb{Z}_{q^{\alpha}}, \frac{x}{q}\right)+O_{q, \alpha}\left(\frac{x(\log \log x)^{j-1}}{(\log x)^{1 /(q-1)}}\right)
\end{aligned}
$$


by Lemma 2.28, Now Theorem 2.27 gives

$$
\begin{array}{r}
D\left(\mathbb{Z}_{q^{\alpha}}, x\right)=C(\boldsymbol{\alpha}) \frac{B_{q}}{q^{\sum_{i=1}^{j} \alpha_{i}}} \frac{x(\log \log x)^{j}}{(\log x)^{1 /(q-1)}}+C(\boldsymbol{\alpha}) \frac{B_{q}}{q^{\sum_{i=1}^{j} \alpha_{i}}} \frac{(x / q)(\log \log (x / q))^{j}}{(\log (x / q))^{1 /(q-1)}} \\
+O_{q, \boldsymbol{\alpha}}\left(\frac{x(\log \log x)^{j-1}}{(\log x)^{1 /(q-1)}}\right)
\end{array}
$$

which establishes the theorem since $(1+1 / q) / q^{\sum_{i=1}^{j} \alpha_{i}}=E_{q}(\boldsymbol{\alpha})$ and $j=\ell(\boldsymbol{\alpha})$.

\section{MAXimally NON-CYCLIC MUlTiPliCATIVE GROUPS-PROOF OF THEOREM 1.6}

Rather than focusing on local Sylow subgroups, we now wish to focus on the global structure of the group $\mathbb{Z}_{n}^{\times}$, and in particular (as described in the introduction) when this group is as far from being cyclic as possible. To define this notion precisely, recall that the primary decomposition of a finite abelian group $G$ is the unique isomorphism of the shape $G \cong \mathbb{Z}_{p_{1}^{r_{1}}} \times \cdots \times \mathbb{Z}_{p_{k}^{r_{k}}}$ where the

$p_{j}^{r_{j}}$ are prime powers (with $r_{j}=1$ possible), while its invariant factor decomposition is the unique isomorphism of the shape $G \cong \mathbb{Z}_{d_{1}} \times \cdots \times \mathbb{Z}_{d_{\ell}}$ where $d_{1}\left|d_{2}\right| \cdots \mid d_{\ell}$.

Definition 3.1. Let $G$ be a finite abelian group of cardinality $m$. We say that $G$ is maximally non-cyclic if any of the four following equivalent conditions hold:

(a) each factor of the primary decomposition of $G$ is of the form $\mathbb{Z}_{p}$ for some prime $p$;

(b) for any prime $p$, the Sylow $p$-subgroup of $G$ is an elementary $p$-group, that is, is of the form $\mathbb{Z}_{p} \times \mathbb{Z}_{p} \times \cdots \times \mathbb{Z}_{p}$

(c) the invariant factors $d_{j}$ are squarefree for every $1 \leq j \leq \ell$;

(d) the largest invariant factor $d_{\ell}$ is minimal among all finite abelian groups of order $m$.

That these four conditions are indeed equivalent is a straightforward exercise in undergraduate algebra. (We remark in passing that it is possible for a finite abelian group to be both cyclic and maximally non-cyclic: such groups are precisely the cyclic groups of squarefree order, which are the orders for which there exists exactly one finite abelian group.)

The most intuitive definitions of maximally non-cyclic are conditions (a)/(b) (which are nearly identical) and condition (d); condition (c), on the other hand, is less intuitive but turns out to be useful in the proof of the characterization of integers whose multiplicative group is maximally non-cyclic. We remark that these equivalent conditions imply that the length $\ell$ of the invariant factor decomposition is maximal among all finite abelian groups of order $m$, although this is not a two-way implication as shown by the examples $\mathbb{Z}_{6} \times \mathbb{Z}_{6}$ (which is maximally non-cyclic) and $\mathbb{Z}_{2} \times \mathbb{Z}_{18}$ (which is not) corresponding to $m=36$ and $\ell=2$.

Our goal in this section is to asymptotically evaluate the counting function for the number of integers $n \leq x$ such that $\mathbb{Z}_{n}^{\times}$is maximally non-cyclic. As in Section 2, we can accomplish this evaluation by giving a characterization of this group-theoretic property in terms of the prime factorization of $n$.

Proposition 3.2. For any $n \in \mathbb{N}$, the group $\mathbb{Z}_{n}^{\times}$is maximally non-cyclic if and only if:

(a) $2^{4} \nmid n$;

(b) $p^{3} \nmid n$ for every odd prime $p$; and

(c) $p-1$ is squarefree for every $p \mid n$. 
Proof. By part (a) of Definition 3.1, together with uniqueness of primary decompositions, a product $H_{1} \times \cdots \times H_{k}$ of finite abelian groups is maximally non-cyclic if and only if each $H_{j}$ is maximally non-cyclic. In particular, $\mathbb{Z}_{n}^{\times}$is maximally non-cyclic if and only if $\mathbb{Z}_{p^{r}}^{\times}$is maximally non-cyclic for every $p^{r} \| n$. Since

$$
\mathbb{Z}_{2^{r}}^{\times} \cong \begin{cases}\text { trivial, } & \text { if } r \leq 1, \\ \mathbb{Z}_{2}, & \text { if } r=2 \\ \mathbb{Z}_{2} \times \mathbb{Z}_{2}, & \text { if } r=3 \\ \mathbb{Z}_{2^{r-2}} \times \mathbb{Z}_{2}, & \text { if } r \geq 4,\end{cases}
$$

we see that $2^{4} \nmid n$ is one necessary condition. Similarly, since for odd primes $p$,

$$
\mathbb{Z}_{\phi\left(p^{r}\right)} \cong \begin{cases}\mathbb{Z}_{p-1}, & \text { if } r=1 \\ \mathbb{Z}_{p} \times \mathbb{Z}_{p-1}, & \text { if } r=2 \\ \mathbb{Z}_{p^{r-1}} \times \mathbb{Z}_{p-1}, & \text { if } r \geq 3\end{cases}
$$

we see that $p^{3} \nmid n$ for all odd primes $p$ is a second necessary condition. Finally, by part (c) of Definition 3.1, the cyclic group $\mathbb{Z}_{p-1}$ is maximally non-cyclic if and only if $p-1$ is squarefree, which is the source of the third and final condition.

Definition 3.3. Let $\xi$ denote Artin's constant

$$
\xi=\prod_{p}\left(1-\frac{1}{p(p-1)}\right)
$$

and define the positive constant

$$
\begin{aligned}
A & =\frac{15}{14 \Gamma(\xi)} \lim _{x \rightarrow \infty}\left\{\prod_{\substack{p \leq x \\
\mu^{2}(p-1)=1}}\left(1+\frac{1}{p}+\frac{1}{p^{2}}\right) \prod_{p \leq x}\left(1-\frac{1}{p}\right)^{\xi}\right\} \\
& =\frac{15}{14 \Gamma(\xi)} \prod_{p}\left(1+\frac{(p+1) \mu^{2}(p-1)}{p^{2}}\right)\left(1-\frac{1}{p}\right)^{\xi} .
\end{aligned}
$$

We will see that the product defining $A$ converges in the proof of Theorem 1.6 below.

It is known that Artin's constant is also the density of the primes $p$ with the property that $p-1$ is squarefree; we provide a proof for the sake of completeness.

Lemma 3.4. For $x \geq 2$, we have $\#\{p \leq x: p-1$ is squarefree $\}=\frac{\xi x}{\log x}+O\left(\frac{x}{\log ^{2} x}\right)$.

Proof. Using the well-known identity $\mu^{2}(n)=\sum_{d^{2} \mid n} \mu(d)$ [6, equation (2.4)], we have

$$
\begin{aligned}
\#\{p \leq x: p-1 \text { is squarefree }\} & =\sum_{p \leq x} \mu(p-1)^{2} \\
& =\sum_{p \leq x} \sum_{d^{2} \mid p-1} \mu(d)=\sum_{d^{2} \leq x} \mu(d) \sum_{\substack{p \leq x \\
d^{2} \mid p-1}} 1=\sum_{d \leq \sqrt{x}} \mu(d) \pi\left(x ; d^{2}, 1\right) .
\end{aligned}
$$


For $d \leq \log ^{2} x$ we use the Siegel-Walfisz theorem [6, Corollary 11.21]

$$
\pi\left(x ; d^{2}, 1\right)=\frac{\operatorname{li}(x)}{\phi\left(d^{2}\right)}+O\left(x e^{-c_{1} \sqrt{\log x}}\right)=\frac{\operatorname{li}(x)}{d \phi(d)}+O\left(\frac{x}{\log ^{4} x}\right),
$$

while for $\log ^{2} x<d \leq \sqrt{x}$ we use the trivial estimate $\pi\left(x ; d^{2}, 1\right) \leq x / d^{2}$. We find that

$$
\begin{aligned}
\sum_{d \leq \sqrt{x}} \mu(d) \pi\left(x ; d^{2}, 1\right) & =\sum_{d \leq \log ^{2} x} \mu(d)\left(\frac{\operatorname{li}(x)}{d \phi(d)}+O\left(\frac{x}{\log ^{4} x}\right)\right)+O\left(\sum_{\log ^{2} x<d \leq \sqrt{x}} \frac{x}{d^{2}}\right) \\
& =\operatorname{li}(x)\left(\sum_{d=1}^{\infty} \frac{\mu(d)}{d \phi(d)}+O\left(\sum_{d>\log ^{2} x} \frac{1}{d \phi(d)}\right)\right)+O\left(\frac{x}{\log ^{2} x}\right) \\
& =\operatorname{li}(x) \prod_{p}\left(1+\frac{\mu(p)}{p \phi(p)}+0+\cdots\right)+O\left(\frac{x}{\log ^{2} x}\right),
\end{aligned}
$$

which implies the statement of the lemma.

The proof of Theorem 1.6 is straightforward if we use the Wirsing-Odoni method; below is a statement of this method [2, Proposition 4] with one hypothesis simplified for our purposes.

Proposition 3.5. Let $f$ be a multiplicative function. Suppose that $0 \leq f\left(p^{r}\right) \leq 1$ for all primes $p$ and all positive integers $r$. Suppose also that there exist real numbers $\omega>0$ and $0<\beta<1$ such that

$$
\sum_{p \leq P} f(p)=\omega \frac{P}{\log P}+O\left(\frac{P}{(\log P)^{1+\beta}}\right)
$$

as $P \rightarrow \infty$. Then the product over all primes

$$
C_{f}=\frac{1}{\Gamma(\omega)} \prod_{p}\left(1+\frac{f(p)}{p}+\frac{f\left(p^{2}\right)}{p^{2}}+\frac{f\left(p^{3}\right)}{p^{3}}+\cdots\right)\left(1-\frac{1}{p}\right)^{\omega}
$$

converges (hence is positive), and

$$
\sum_{n \leq N} f(n)=C_{f} N(\log N)^{\omega-1}+O_{f}\left(N(\log N)^{\omega-1-\beta}\right)
$$

as $N \rightarrow \infty$.

Proof of Theorem 1.6. Fix $0<\varepsilon<1$. Let $f$ be the indicator function of the set of integers $n$ with the property that $\mathbb{Z}_{n}^{\times}$is maximally non-cyclic. By Proposition 3.2, the function $f$ is multiplicative with values on prime powers

$$
f\left(p^{r}\right)= \begin{cases}1, & \text { if } p=2 \text { and } 1 \leq r \leq 3 \\ 1, & \text { if } p \geq 3 \text { and } 1 \leq r \leq 2 \text { and } p-1 \text { is squarefree } \\ 0, & \text { otherwise. }\end{cases}
$$

By Lemma 3.4, this function satisfies the hypotheses of Proposition 3.5 with $\omega=\xi$ and $\beta=1-\varepsilon$. We conclude from that proposition that

$$
\#\left\{n \leq x: \mathbb{Z}_{n}^{\times} \text {is maximally non-cyclic }\right\}=\frac{A x}{(\log x)^{1-\xi}}+O_{\varepsilon}\left(\frac{x}{(\log x)^{2-\xi-\varepsilon}}\right),
$$


where $A$ is given by the convergent product

$$
\begin{aligned}
A & =\frac{1}{\Gamma(\xi)} \prod_{p}\left(1+\frac{f(p)}{p}+\frac{f\left(p^{2}\right)}{p^{2}}+\frac{f\left(p^{3}\right)}{p^{3}}+\cdots\right)\left(1-\frac{1}{p}\right)^{\xi} \\
& =\frac{1}{\Gamma(\xi)}\left(1+\frac{1}{2}+\frac{1}{4}+\frac{1}{8}\right) \lim _{x \rightarrow \infty}\left(\prod_{\substack{3 \leq p \leq x \\
p-1 \text { squarefree }}}\left(1+\frac{1}{p}+\frac{1}{p^{2}}\right) \prod_{2 \leq p \leq x}\left(1-\frac{1}{p}\right)^{\xi}\right) \\
& =\frac{15}{14 \Gamma(\xi)} \lim _{x \rightarrow \infty}\left(\prod_{\substack{2 \leq p \leq x \\
p-1 \text { squarefree }}}\left(1+\frac{1}{p}+\frac{1}{p^{2}}\right) \prod_{2 \leq p \leq x}\left(1-\frac{1}{p}\right)^{\xi}\right)
\end{aligned}
$$

as claimed.

\section{REFERENCES}

[1] Ben Chang and Greg Martin. The smallest invariant factor of the multiplicative group. Int. J. Number Theory (to appear).

[2] Steven Finch, Greg Martin, and Pascal Sebah. Roots of unity and nullity modulo n. Proc. Amer. Math. Soc., 138(8):2729-2743, 2010.

[3] Kevin Ford, Florian Luca, and Pieter Moree. Values of the Euler $\phi$-function not divisible by a given odd prime, and the distribution of Euler-Kronecker constants for cyclotomic fields. Math. Comp., 83(287):1447-1476, 2014.

[4] Edmund Landau. Über die Einteilung der positiven ganzen Zahlen in vier Klassen nach der Mindestzahl der zu ihrer additiven Zusammensetzung enforderlichen Quadrate. Arch. der Math. und Phys., 13(3):305-312, 1908.

[5] Greg Martin and Lee Troupe. The distribution of the number of subgroups of the multiplicative group. J. Aust. Math. Soc., 108(1):46-97, 2020.

[6] Hugh L. Montgomery and Robert C. Vaughan. Multiplicative number theory. I. Classical theory, volume 97 of Cambridge Studies in Advanced Mathematics. Cambridge University Press, Cambridge, 2007.

Department of Mathematics and Statistics, C245 (Science Building), 1000 KLO Road, Kelowna, BC, CANADA V1Y 4X8

E-mail address: jdowney@okanagan.bc.ca

Department of Mathematics, University of British Columbia, Room 121, 1984 Mathematics Road, VANCOUVER, BC, CANADA V6T $1 \mathrm{Z2}$

E-mail address: gerg@math.ubc.ca 